\title{
Reflection in Seyfert galaxies and the unified model
}

\author{
Roland WALTER* \\ INTEGRAL Science Data Centre, Université de Genève, Chemin d'Ecogia 16, CH-1290 Versoix, \\ Switzerland \\ E-mail: Roland.Walter@unige.ch

\section{Claudio RICCI} \\ INTEGRAL Science Data Centre, Université de Genève, Chemin d'Ecogia 16, CH-1290 Versoix, \\ Switzerland
}

\begin{abstract}
We present a study of the average hard X-ray spectra of Seyfert galaxies accumulating $100 \mathrm{Ms}$ of INTEGRAL observing time. Our aim is to test the unified model of Active Galactic Nuclei, and to constrain differences and similarities between different classes of objects. We analysed all public INTEGRAL IBIS/ISGRI data available on 165 Seyfert galaxies detected at $\mathrm{z}<0.2$. Our final sample consists of 44 Seyfert 1, 29 Seyfert 1.5, 78 Seyfert 2, and 14 Narrow Line Seyfert 1. For each subsample, we stacked all the images, and derived their average hard X-ray spectra in the 17-250 keV energy range. All classes of Seyfert galaxies show on average the same nuclear continuum, as foreseen by the zeroth order unified model. The average spectrum of midly obscured Seyfert $2 \mathrm{~s}$ is different, featuring a broad excess of emission at $30 \mathrm{keV}$ that we interpret as a strong reflection component. This correlation between reflection and absorption suggests that the Seyfert type is determined by the environment rather than by inclination. Furthermore this large reflection component might reduce the amount of Compton-thick objects needed to explain the peak of the cosmic X-ray background. A simple fit to the CXB shows that our findings might imply that the fraction of Compton-thick sources is not larger than $10 \%$.
\end{abstract}

The Extreme and Variable High Energy Sky - extremesky2011,

September 19-23, 2011

Chia Laguna (Cagliari), Italy

\footnotetext{
* Speaker.
} 


\section{Introduction}

The orientation-based unified model [1] is successful at explaining several features of the Active Galactic Nuclei (AGN) population. This model is however a generalisation, a simplification of a complex reality and suffers from several difficulties: a small fraction of Seyfert 1 galaxies are absorbed (e.g. [2]), some Seyfert 2 galaxies do not show any X-ray absorption (e.g. [3]) and $50-70 \%$ of Seyfert 2 galaxies miss polarised broad lines [4, 5, 6].

The analysis of recent high resolution (VLTI, Gemini) infrared observations of nearby AGNs confirmed the presence of a geometrically thick, torus-like dust distribution [7, 8, 9]. However these studies revealed the presence of irregular and clumpy tori. The classification of a Seyfert galaxy as a type 1 or 2 was found to depend more on the intrinsic properties of the tori (in particular the light escape probability) rather than on their mere inclination. Recent X-ray observations provided similar indications that the absorbing torus features properties that are not linked with inclination:

- Eguchi et al $(2009,2011)[10,11]$ analysed Suzaku observations of new Swift/BAT AGNs and found that these objects are of a new type, featuring low scattering and strong reflection that cannot be explained by orientation effect but by a different torus geometry.

- Miller et al (2009) and Lobban et al (2011) [12,13] found that the long term variability of NGC 4051, observed by Suzaku, is best interpreted by a significant change in the covering fraction of a Compton thick partial covering material obscuring the emission from the central engine.

- Risaliti et al (2009) [14] detected a variable partial covering cloud in NGC 1365 crossing the line of sight in $25 \mathrm{ksec}$. Subsequent Suzaku observations suggested a complex structure of the circum-nuclear medium, consisting of at least two distinct components with rather different physical properties, one of them covering more than $80 \%$ of the continuum source with a column density of (3-4) $10^{24} \mathrm{~cm}^{-2}$.

\section{INTEGRAL stacking analysis}

We selected all 205 Seyfert galaxies detected at hard X-rays by INTEGRAL IBIS/ISGRI during its first 8 years of operations. We excluded a number of sources because they were too close to other sources, had an unclear classification or a too large redshift or because they were detected with a too large significance. The final sample used for the analysis consists of 165 AGN, of these 44 are Seyfert 1, 29 Seyfert 1.5, 68 Compton-thin Seyfert 2, 10 Compton thick Seyfert 2, and 14 NLS1. The Seyfert 2 galaxies were further separated in 35 lightly $\left(N_{H}<10^{23} \mathrm{~cm}^{-2}\right), 27$ midly $\left(10^{23}>N_{H}>10^{24} \mathrm{~cm}^{-2}\right)$ and 10 highly $\left(N_{H}>10^{24} \mathrm{~cm}^{-2}\right)$ obscured subsamples.

We stacked the spectra of the sources for each subsample, accumulating up to $44 \mathrm{Msec}$ of observing time for Seyfert 2 galaxies and obtained high signal-to-noise average spectra for every sub-class. We then compared the spectra in model independent and model dependent ways. The results are as follow [15]:

- Seyfert 1 and Seyfert 1.5 have identical hard X-ray spectra with average photon index of $1.8 \pm 0.2$, cutoff energy of $>190 \mathrm{keV}$ and reflection parameter of $0.2 \pm 0.2$. 
- Seyfert 2 have completely different spectra, on average, featuring a broad bump below 50 $\mathrm{keV}$.

- The average spectrum of lightly obscured Seyfert 2 is completely consistent with those of Seyfert 1 and 1.5. Their average reflection parameter is $<0.5$ (Fig. 2).

- The average spectrum of midly obscured Seyfert 2 (Fig. 1) features a strong and broad excess below $50 \mathrm{keV}$ and is best represented by a photon index of $1.8 \pm 0.1$, a cutoff energy $<400$ $\mathrm{keV}$ and a reflection parameter of $2.2_{-1}^{+4}$ ( $3 \sigma$ uncertainties). A bi parametric analysis of the cutoff energy vs the reflection parameter indicates that the latter is $>1$ with a confidence larger than 99\% (Fig. 2 ).

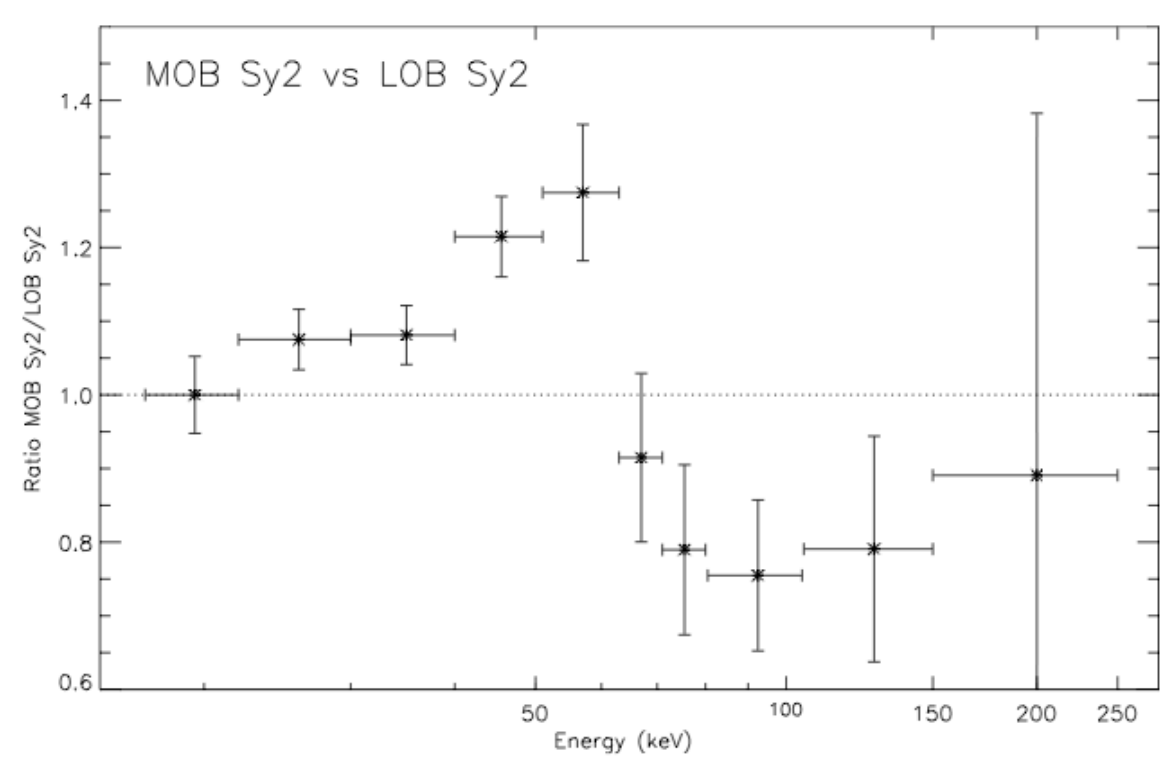

Figure 1: The ratio between the count spectra of midly and lightly obscured Seyfert 2 galaxies.

\section{Discussion}

The stacking analysis of INTEGRAL spectra of Seyfert galaxies indicates that (1) the intrinsic $\mathrm{X}$-ray continuum of Seyfert galaxies is consistent with an average Compton optical depth of $0.8 \pm$ 0.5 for all classes of objects; (2) the average cutoff energy ( $>190 \mathrm{keV}$ ) is consistent with the results derived from BeppoSax [16]; (3) the average reflection in Seyfert 1 and lightly obscured Seyfert 2 galaxies $(\mathrm{R}<0.5)$ is consistent with that expected from an accretion disk; and (4) the average reflection in midly obscured Seyfert 2 galaxies is not physical, unless their continuum emission is attenuated by a factor larger than two.

The correlation we have found between reflection and absorption points towards a clear change in the environment of Seyfert galaxies. The emerging picture is that obscuring/reflecting clouds have on average a lower covering factor in Seyfert 1 than in Seyfert 2 galaxies. This indicates that 


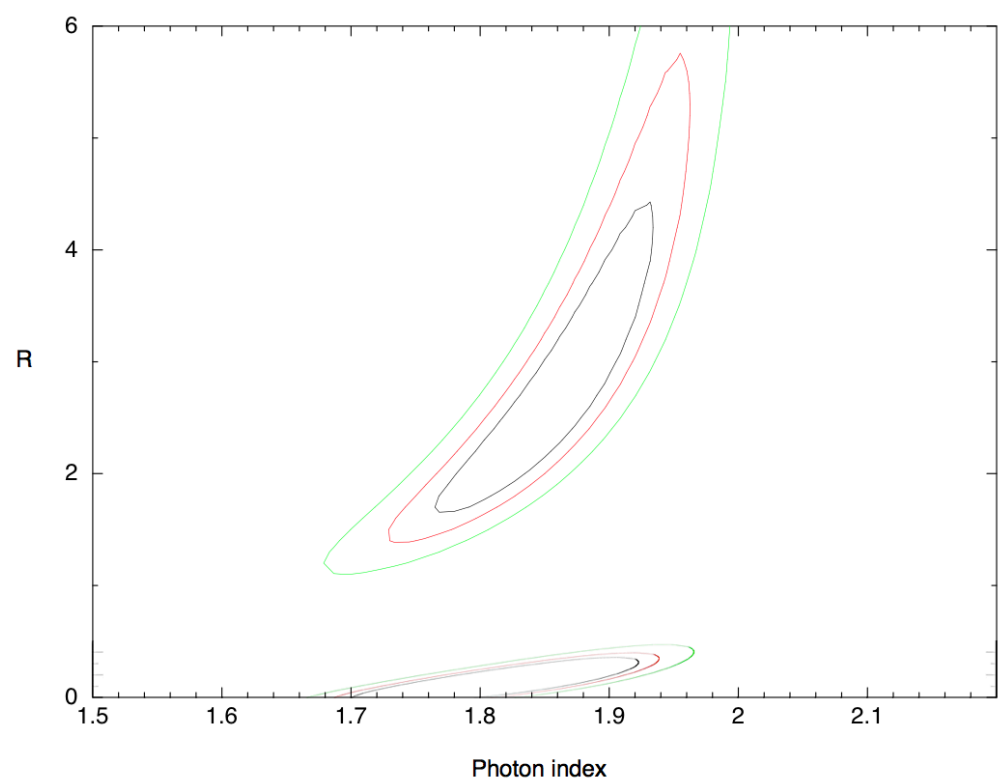

Figure 2: Multi-parametric contour plot providing the 68, 90 and $99 \%$ confidence level on the power law photon index $\Gamma$ and the reflection parameter $R$ for the average spectrum of midly $\left(10^{23}<N_{H}<10^{24} \mathrm{~cm}^{-2}\right.$; top) and lightly $\left(N_{H}<10^{23} \mathrm{~cm}^{-2}\right.$; bottom) obscured Seyfert 2 .

the Seyfert type is driven by the environment rather than by orientation. A conclusion very similar to these obtained from recent infrared and X-ray observations.

To synthesize the spectrum of the cosmic X-ray background (CXB), Gilli et al. (2007) [17] needed to assume that about $30 \%$ of the AGN population should be Compton thick to explain the CXB excess at $30 \mathrm{keV}$. This population is however hardly detected. INTEGRAL observations indicate that midly obscured Seyfert 2 galaxies have much stronger reflection in the local Universe. Extrapolating this at high redshift provides a new picture, where the CXB can be explained with only $10 \%$ of Compton thick active galactic nuclei, a fraction that corresponds much better to the current survey observations.

\section{References}

[1] Antonucci, R. R. J. and Miller, J. S., ApJ 297 (1985) 621.

[2] Cappi, M. et al., A\&A 446 (2006) 459.

[3] Panessa, F. and Bassani, L., A\&A 394 (2002) 435.

[4] Tran, H. D., ApJ 554 (2001) L19.

[5] Tran, H. D., ApJ 583 (2003) 632.

[6] Gu, Q. and Huang, J., ApJ 579 (2002) 205.

[7] Jaffe, W. et al., Nature 429 (2004) 47.

[8] Tristram, K. R. W. et al., A\&A 474 (2007) 837. 
[9] Ramos Almeida, C. et al., ApJ 731 (2011) 92.

[10] Eguchi, S., Ueda, Y., Terashima, Y., Mushotzky, R., and Tueller, J., ApJ 696 (2009) 1657.

[11] Eguchi, S. et al., ApJ 729 (2011) 31.

[12] Miller, L. et al., MNRAS 403 (2010) 196.

[13] Lobban, A. P. et al., MNRAS 414 (2011) 1965.

[14] Risaliti, G. et al., ApJ 705 (2009) L1.

[15] Ricci, C., Walter, R., Courvoisier, T. J.-L., and Paltani, S., A\&A 532 (2011) A102.

[16] Dadina, M., A\&A 485 (2008) 417.

[17] Gilli, R., Comastri, A., and Hasinger, G., A\&A 463 (2007) 79. 